\title{
Ionic Liquid Matrix-Induced Metastable Decay of Peptides and Oligonucleotides and Stabilization of Phospholipids in MALDI FTMS Analyses
}

\author{
Jeffrey J. Jones, S. Mariccor A. B. Batoy, and Charles L. Wilkins \\ Department of Chemistry and Biochemistry, University of Arkansas, Fayetteville, Arkansas, USA
}

\author{
Rohana Liyanage and Jackson O. Lay, Jr. \\ Department of Chemistry and Biochemistry, University of Arkansas Statewide Mass Spectrometry Facility, \\ Fayetteville, Arkansas, USA
}

\begin{abstract}
Room-temperature ionic liquid matrices (ILMs) have recently been investigated for use in matrix-assisted laser desorption/ionization (MALDI) mass spectrometry (MS) and proven to be advantageous. Literature accounts of ILM performance for biological samples document increased sensitivity and ionization efficiency. These claims have been investigated here, and are supported for MALDI TOF applications to peptides, oligonucleotides, and phospholipids. Peptides and oligonucleotides however, do not behave in the same way when ILMs are used for MALDI FTMS. As reported here, with 3 tesla MALDI FTMS peptides and oligonucleotides fragment readily. These observations contrast with those found for MALDI time-of-flight mass spectrometry. Fragmentation is apparently slower than the time required to accelerate ions in a MALDI TOF mass spectrometer, but is readily observed by MALDI FTMS. Therefore, fragmentation of these molecules must occur on a relatively slow time scale. As trapping time is extended, increased fragmentation of peptides and oligonucleotides is seen. However, phospholipids do not fragment extensively. Furthermore, use of traditional solid matrices causes significant fragmentation for this category of compound but is suppressed by use of ILMs. (J Am Soc Mass Spectrom 2005, 16, 2000-2008) (c 2005 American Society for Mass Spectrometry
\end{abstract}

S ince its inception, matrix assisted laser desorption/ionization has been applied with great success to a number of biological and synthetic polymers [1, 2]. MALDI mass spectrometry has been especially useful in analytical biochemistry, affording researchers the ability to study biological compounds isolated from their environments [3] or with minimal purification [4-15]. This characteristic separates MALDI from the other major soft ionization technique of electrospray ionization, which requires extensive control over solvent conditions to optimize analyte ionization. MALDI, and perhaps the recentlydeveloped desorption electrospray ionization method (DESI) [16], are amongst the few ionization techniques that can be used to study whole cells and tissue samples directly, affording researchers the ability to compare spectral profiles for identification or to image surfaces for characteristic compounds [4-15]. The attributes responsible for the success of

Published online October 24, 2005

Address reprint requests to Dr. C. L. Wilkins, Department of Chemistry and Biochemistry, University of Arkansas, Fayetteville, AR 72701, USA. E-mail: cwilkins@comp.uark.edu
MALDI matrices in biochemical analysis are the propensity to absorb laser energy and to promote ionization of polar analyte molecules [1, 2, 17]. MALDI matrices are selected because they have UV chromophores at a desired wavelength, matching the desorption laser properties. They are often small acidic organic molecules that can cocrystallize with the analyte and provide an accessible source of protons. An ideal matrix would also provide a homogeneous and reproducible sample. This is rarely achieved for solid matrices [18]. Liquid matrices have been employed in the past with great success, for example in fast atom bombardment mass spectrometry [19]. Such matrices have the advantage of promoting sample homogeneity as well as continuously replacing desorbed material as it is ablated from the surface. Despite these advantages, liquid matrices have found limited success in UV MALDI applications.

In recent studies, room temperature ionic liquid matrices (ILMs) have been investigated for their use in MALDI mass spectrometry. This research clearly demonstrated enhanced ionization efficiencies and improved spot to spot reproducibility [20-24]. The ILMs 
discussed here are analogs of the successful solid matrices, chosen because of the high quality purified protein, oligonucleotide, and phospholipid spectra obtained using MALDI TOF mass spectrometers [22-24]. In view of the demonstrated advantages, it was considered worthwhile to investigate the use of ILMs for MALDI FTMS. The present study shows that use of ILMs increased the sensitivity of MALDI TOF and FTMS both for pure compounds and complex biological samples. In addition, it is found that for peptides and oligonucleotides, increased fragmentation yielding information complementary to MALDI TOF data is available by MALDI FTMS.

\section{Experimental}

\section{Materials}

The peptides bradykinin, substance-P, melittin, allatostatin IV, and the organic acids 2,5 dihydroxy benzoic acid (DHB), $\alpha$-cyano-4-hydroxy-cinnamic acid (CHCA) and 3-hydroxypicolinic acid (3HPA) used in this study were purchased from SigmaAldrich Co. (St. Louis, MO) and used without further purification. Aniline was purchased from Alfa Aesar (Ward Hill, MA), N,N-diethylaniline from Alfa Aesar Lancaster (Pelham, NH), and $n$-butylamine from TCI America (Portland, OR) at purities greater than $99 \%$. The oligonucleotide 5'-GGATTC-3' was purchased from Sigma Genosys (The Woodlands, TX) and used without further purification. Phosphatidylcholine, L- $\alpha$ phosphatidylcholine- $\beta$-palmitoyl-oleoyl, ([PC 16:0, 18:1]) $5 \mathrm{mg}$ dry sample, and phosphatidylethanolamine, 1-2,dioleoyl-sn-glycerol-3-phospho-ethanolamine, ([PE $18: 1,18: 1]) 2.5 \mathrm{~mL}$ of a $10 \mathrm{mg} / \mathrm{mL}$ sample in trichloromethane were purchased and used without further purification (Sigma-Aldrich Co., St. Louis, MO). The phosphatidylcholine was suspended in $500 \mu \mathrm{L}$ of trichloromethane to make a $10 \mathrm{mg} / \mathrm{mL}$ solution. All analytes were diluted to a concentration of $5.0 \mu \mathrm{Mol}$ for analysis, and spotted in $1 \mu \mathrm{L}$ aliquots to a $1 \mu \mathrm{L}$ matrix spot of an ILM, allowing the analyte solvent to evaporate before analysis. For solid matrices, a $1 \mu \mathrm{L}$ aliquot of the analyte was added to a $0.5 \mu \mathrm{L}$ spot of solid matrix, allowed to dry and then followed by another $0.5 \mu \mathrm{L}$ of solid matrix solution. Fragmentation prediction was simulated in silico using MS-product from Protein Prospector v4.0.5 (http://prospector.ucsf.edu/) and theoretical isoelectric points were calculated using ExPASy's Compute pI/MW tool (au.expasy.org/tools/ pi_tool.html). In silico fragmentation for the oligonucleotide was carried out using the "DNA Fragment Calculator" authored by David L. Tabb, and provided on line under the GPL at (fields.scripps.edu/DFCalc/ index.html). The Java source code was modified and recompiled to allow calculations using monoisotopic masses.

A few ionic liquid matrices reported as successful in biological analysis were synthesized as described in literature [20-22, 24]. The following ILMs were synthesized for study with MALDI FTMS: $n$-butylamine salt of CHCA (nBA-CHCA), N,N-diethylaniline salt of CHCA (DEA-CHCA), aniline salt of CHCA (A-CHCA), N,Ndiethylaniline salt of DHB (DEA-CHCA), and n-butylamine salt of DHB (nBA-DHB). Briefly, $0.5 \mathrm{~g}$ of CHCA or DHB was dissolved in $15 \mathrm{~mL}$ of methanol, to which an equimolar amount of base was added. The solution was stirred for five or more minutes and then placed on a rotary-evaporator to remove the methanol. $N, N$-diethylaniline-CHCA and N,N-diethylanilineDHBA are liquid at room temperature and at $1 \times 10^{-9}$ torr. Aniline-CHCA was a liquid for $10 \mathrm{~min}$ at room temperature immediately following methanol evaporation, then became solid. For use in MALDI experiments, $0.25 \mathrm{~mL}$ of methanol was added to $\sim 0.75 \mathrm{~g}$ of ILM material and spotted the same as a traditional solid matrix. The ILM $n$-butylamine-CHCA became a solid at room temperature, $\sim 10 \mathrm{~min}$ after evaporation of the methanol solution. However, if $\sim 0.75 \mathrm{~g}$ of solid nBACHCA is suspended in $100 \mu \mathrm{L}$ of methanol, the solution is somewhat viscous and can be spotted in $1 \mu \mathrm{L}$ aliquots that maintain a liquid appearance at room temperature and pressure as well as at $1 \times 10^{-9}$ torr.

\section{Data Acquisition and Analysis}

MALDI FTMS spectra were collected with a 3 tesla FTMS (IonSpec Corp., Lake Forest, CA) utilizing a nitrogen laser operating at $\lambda=337 \mathrm{~nm}$ at full power $(60-70 \mu \mathrm{J})$. Laser power was measured using a Molectron Detector, Inc., model EPM2000 Joule meter (Portland OR). The laser spot size is measured to be about $0.196 \mathrm{~mm}^{2}$. The instrument utilizes a $100 \mathrm{msec}$ pulse on an inlet valve to allow a reservoir of nitrogen gas at a pressure of 40 torr to cool the desorbed ions. In some cases, an ion accumulation strategy was employed to increase signal strength. This procedure used three laser pulses for ion accumulation followed by a single excitation and detection. In most instances, using the ILM as a MALDI matrix, a single desorption event was more than adequate compared to experience with solid crystalline matrices that sometimes require use of three laser pulses to boost signal strength. Background pressure measured in the source region during signal acquisition was typically $1 \times 10^{-8}$ torr to $1 \times 10^{-9}$ torr. The evacuated analyzer region of the instrument is separated from the source region by a $5 \mathrm{~mm}$ diameter conductance limit and typically measures a vacuum pressure of $5 \times 10^{-11}$ torr.

MALDI TOF spectra were collected with a Bruker Reflex III (Bruker, UK), in reflectron mode, utilizing a nitrogen laser operating at $\lambda=337 \mathrm{~nm}$ at between 3 and $20 \%$ full power (53-70 $\mu \mathrm{J}$, respectively). Laser power was measured using a Molectron Detector, Inc., model EPM2000 Joule meter (Portland OR). The laser spot size is measured to be about $0.071 \mathrm{~mm}^{2}$. Background pressure during signal acquisition was typically $5.5 \times 10^{-8}$ torr to $7.5 \times 10^{-8}$ torr. All calibrations were calculated 

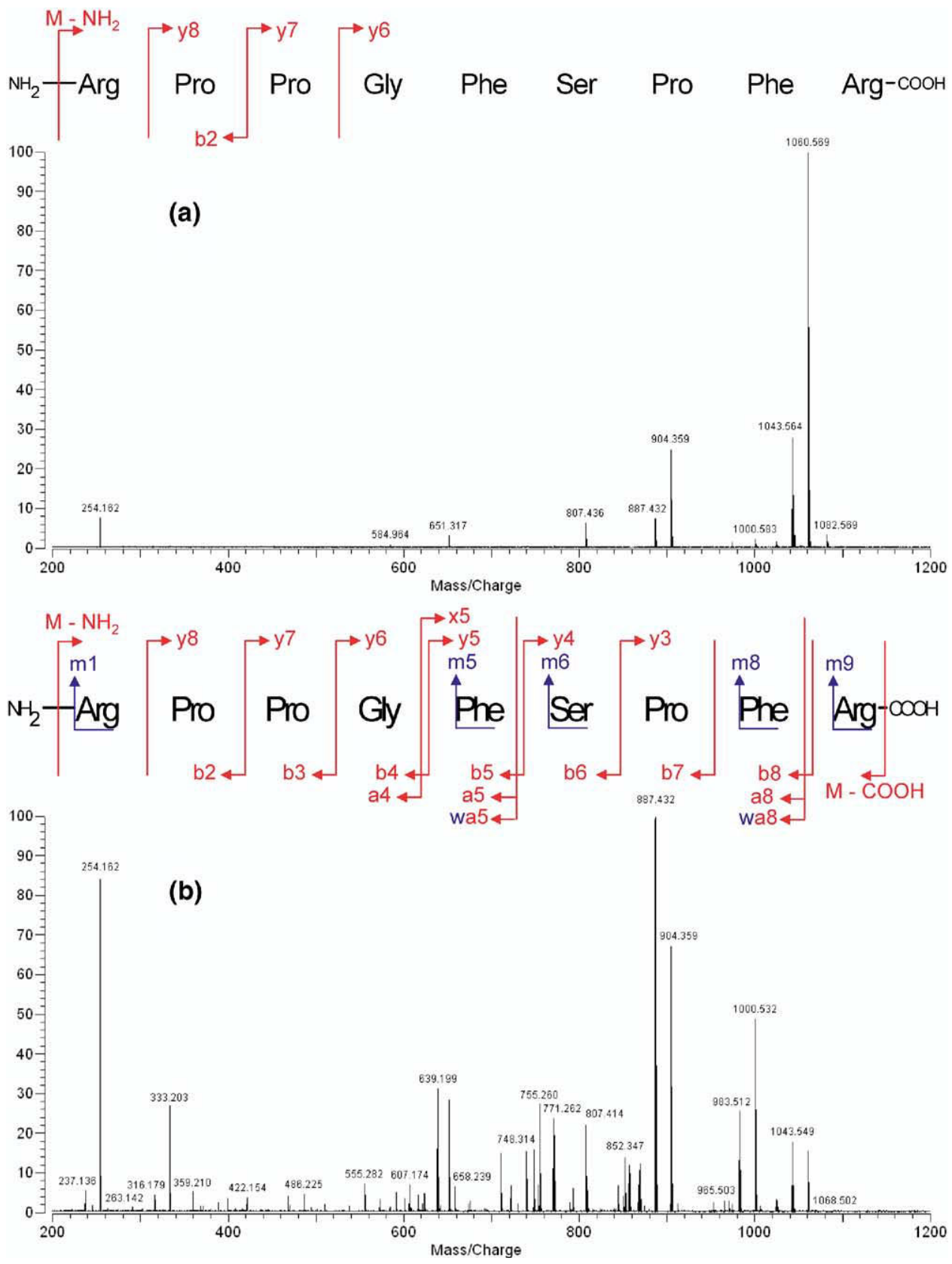

Figure 1. MALDI FTMS spectra of bradykinin collected with anexternal source 3 Tesla FTMS using a nitrogen laser $(\lambda=337 \mathrm{~nm})$, (a) with DHB used as the matrix, (b) with nBA-CHCA used as the matrix.

using an external polyethylene glycol 1500 spectrum collected during the experiments and matched to the total ion intensity. Mass measurement accuracy for these experiments was $\sim 10$ ppm.

An attempt was made to compare CHCA derived ILMs with solid CHCA, however solid CHCA did not produce adequate peptide or oligonucleotide spectra at the maximum laser output available for this study. Therefore, ILMs were compared with solid DHB, a trend that is consistent in current literature. ILMs derived from DHB were also synthesized and tested; the DHB ILMs also did not produce spectra at the maximum laser output available for this study.

\section{Results and Discussion}

MALDI TOF spectra for each of four peptides spotted with each of the five ILMs were collected at a laser power just above the threshold for desorption/ionization (vide infra). The laser power measured for each of these ranged from 53 to $70 \mu \mathrm{J}$, and a total of 70 measurements was summed to give a final spectrum. The ionic liquid matrix DEA-DHB yielded the best results in terms of having the highest total ion count among each of the matrices and for all four peptides. The ionization threshold for this ILM is estimated at 60 $\mu \mathrm{J}$, and although this matrix produced the greatest ion 

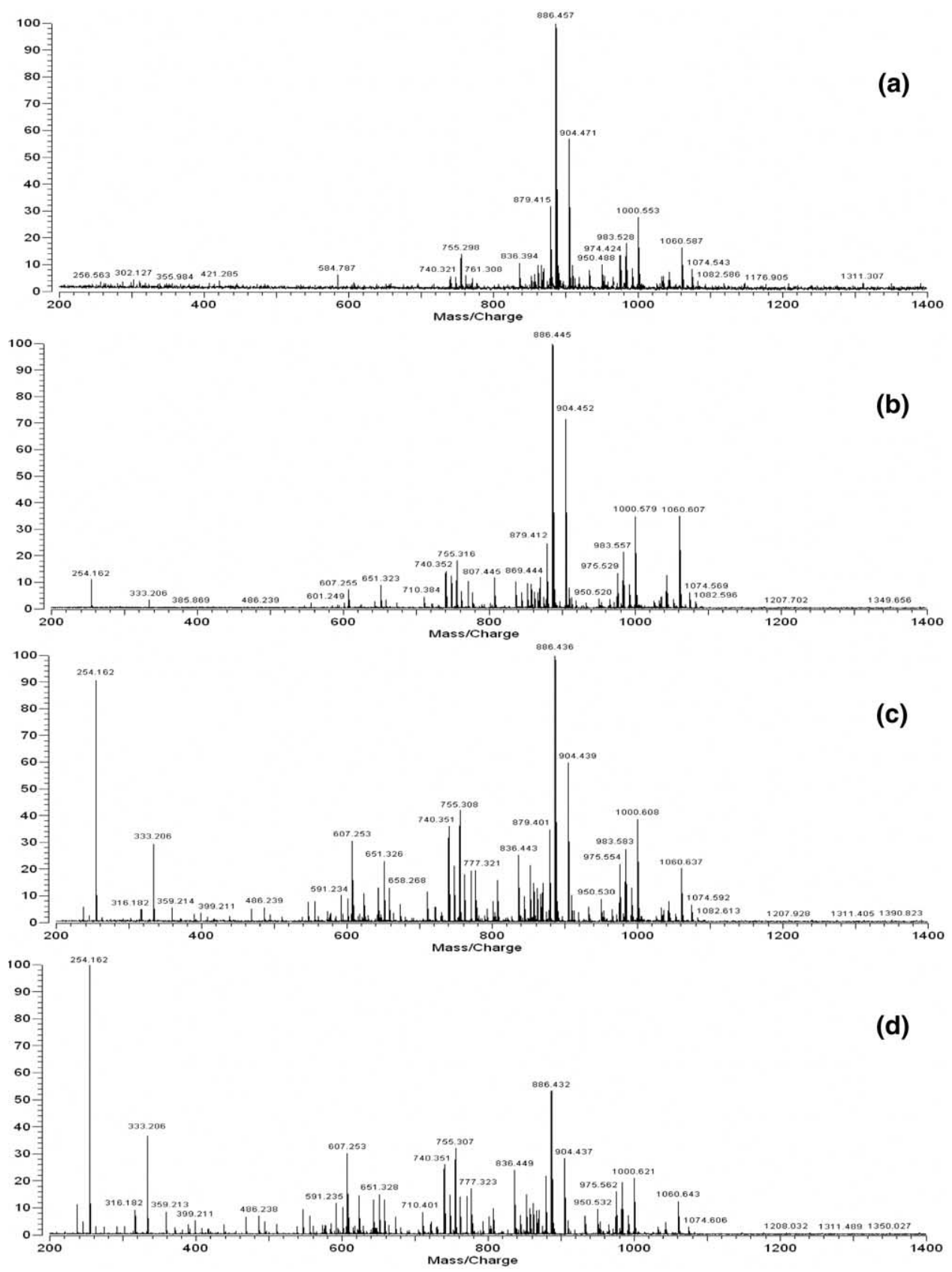

Figure 2. MALDI FTMS spectra of bradykinin, spotted with nBA-CHCA, collected after increasing ion storage duration with an external source 3 Tesla FTMS using a nitrogen laser $(\lambda=337 \mathrm{~nm})$. Externally generated MALDI ions are transported to the ICR cell via a quadrupole ion guide and are trapped $788 \mu \mathrm{sec}$ after the initial laser pulse. The ions were then stored in the ICR cell for (a) $1.0 \mathrm{~s},(\mathbf{b})$ $3.0 \mathrm{~s}$, (c) $7.5 \mathrm{~s}$, and (d) $15.0 \mathrm{~s}$, prior to observation.

abundances for each of the samples, the ILM DEA$\mathrm{CHCA}$ required only $53 \mu \mathrm{J}$ to produce nearly the same results. Overall, it is observed that ILMs increase the ionization efficiency as reported previously [20-22, 24], and increase the shot-to-shot reproducibility for molecules analyzed by TOF, also previously reported [22]. Although present measurements by MALDI TOF are consistent with previous reports, use of ILMs has not been previously investigated by MALDI FTMS, and in this application, the ILMs show somewhat different behavior than expected, based only on prior MALDI TOF results.

The ionic liquid matrix MALDI FTMS spectra of each of the four peptides desorbed at the ionization threshold with a single nitrogen laser pulse reveal results that are remarkably different from spectra collected using a solid matrix. Here, as mentioned above, laser power 60-70 $\mathrm{J}$ was used. Figure 1 clearly shows the difference between solid and liquid MALDI FTMS (in this and other spectra, absolute intensity and resolving 

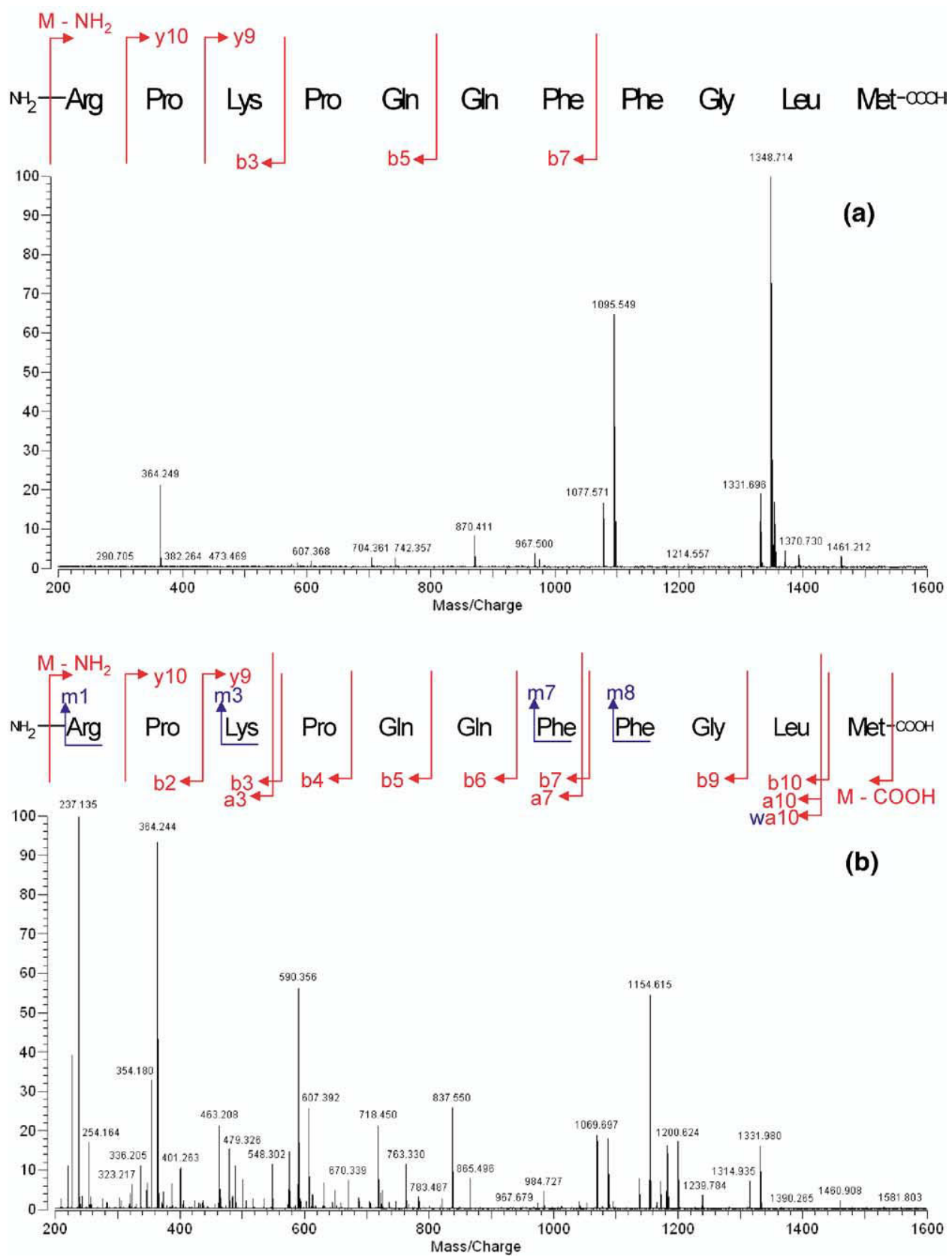

Figure 3. MALDI FTMS spectra of substance $P$ collected with an external source 3 Tesla FTMS using a nitrogen laser $(\lambda=337 \mathrm{~nm})$, (a) with DHB used as the matrix, (b) with DEA-CHCA used as the matrix.

power for the base peak is included for ease of comparison). The top spectrum of the protein bradykinin was collected using DHB as the matrix. The parent molecular mass is the dominant ion detected, with very little fragmentation occurring. The bottom spectrum in Figure 1 is of the same sample with identical instrumental parameters. However, DEA-CHCA was used as the matrix. These data suggest that the ILM transfers a greater amount of laser energy to the sample analyte, allowing for more thermal degradation. It is important to note that although ILMs increase the amount of protein fragmentation in FTMS, the sensitivity of the measurement has also increased. Noted in Figure 1 is the type and extent of fragmentation observed in MALDI FTMS. For solid matrices, the small amount of fragmentation observed occurs along the protein backbone, producing a few $\mathrm{y}$ and $\mathrm{b}$ ions, with a lesser amount of water or ammonia loss. Ionic liquid matrices, on the other hand, promote complete backbone cleavage, giving rise to all but the smallest $y$ and $b$ ions, a few 'a and ' $x$ ions, multiple backbone cleavages resulting in internal ladder sequences, functional group losses for every amino acid possible, and extensive water and ammonia loss. Clearly, there is an extremely energetic 


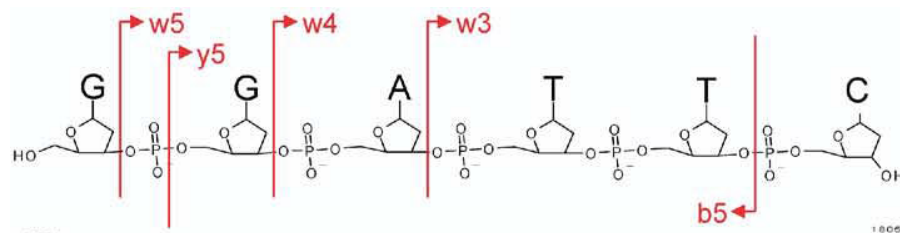

(a)
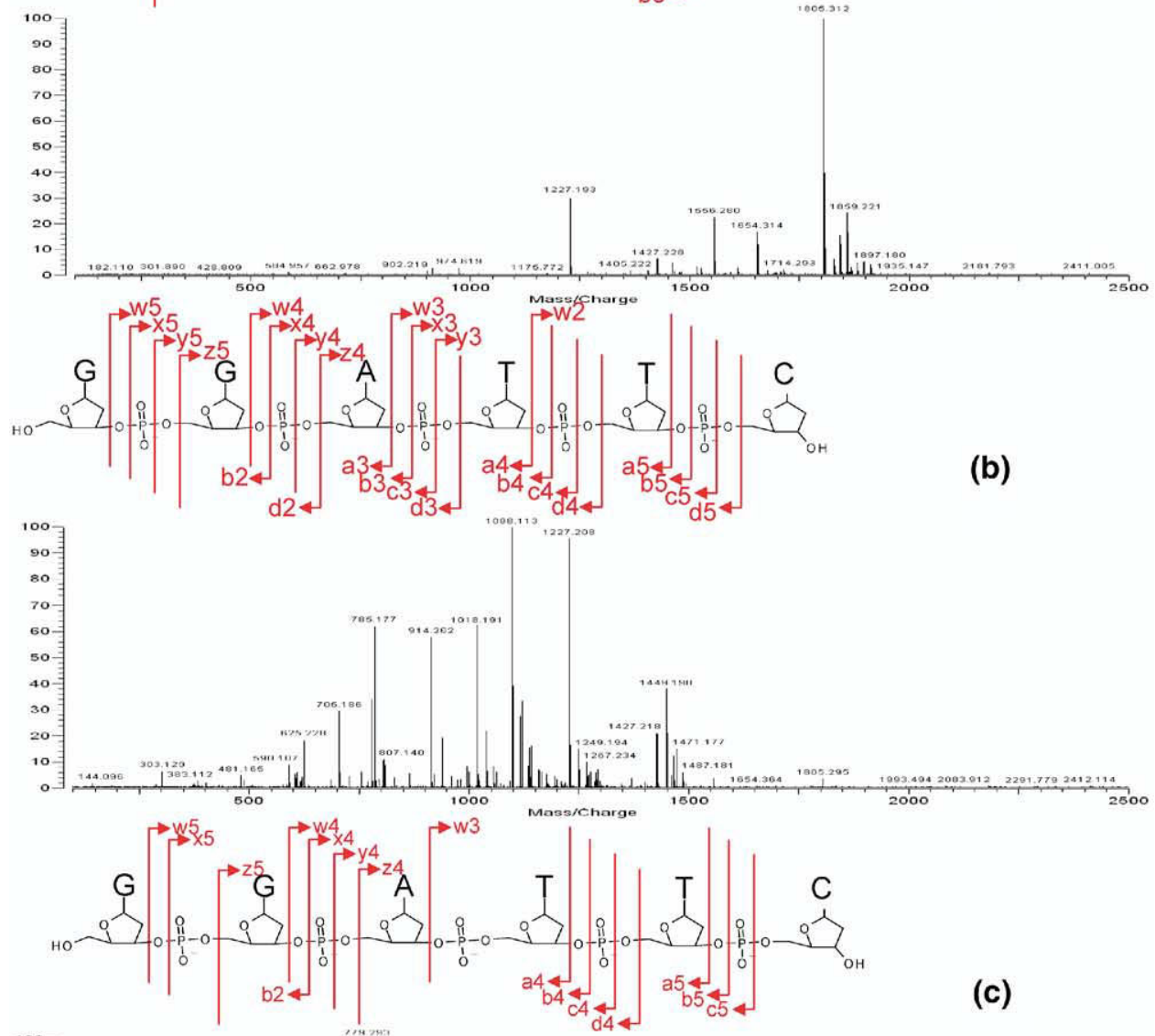

(b)

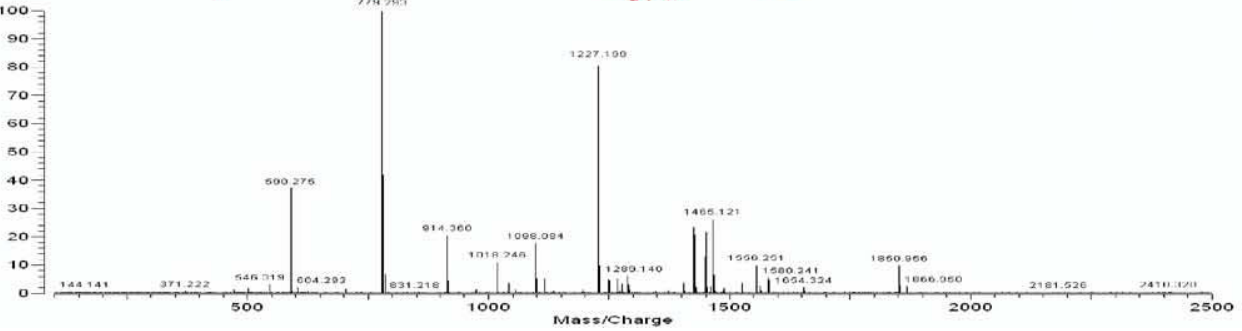

Figure 4. MALDI FTMS spectra of the oligonucleotide 5'-GGATTC-3' analyzed using (a) 3HPA as the matrix, (b) A-CHCA as the matrix, (c) DEA-CHCA as the matrix.

process occurring that traditional solid matrices such as DHB dampen. ILMs must, therefore, have a different mechanism of action, most likely attributable to their aprotic nature.

The time required for transfer of ions from the external source to the observation cell is at least 788 $\mu \mathrm{s}$, enough time for ions to begin to dissociate because of excess kinetic energy (Figure 1b). By extending the delay between ion trapping and chirp excitation, ions are found to decay further in the FTMS cell. At vacuum pressures lower than $5 \times 10^{-11}$ torr for the longer delays, magnetron collision induced dissociation can be ruled out. Fragmentation of peptides by MALDI FTMS through the use of ILMs rivals both SORI CAD [25] and IRMPD [26] in the number and extent of fragmentation. Figure 2 is a good indication that the process for fragmentation is longer than expected for prompt fragmentations. It is sufficiently slow so that full dissociation occurs only after $3000 \mathrm{~ms}$.

The MALDI FTMS spectrum of Substance P, Figure 3 , reveals the same type of fragmentation as for bradykinin. Bradykinin and Substance $\mathrm{P}$ have very similar calculated isoelectric points and would be expected to fragment in much the same way. Substance $\mathrm{P}$, however, is observed with a majority of $b$ 


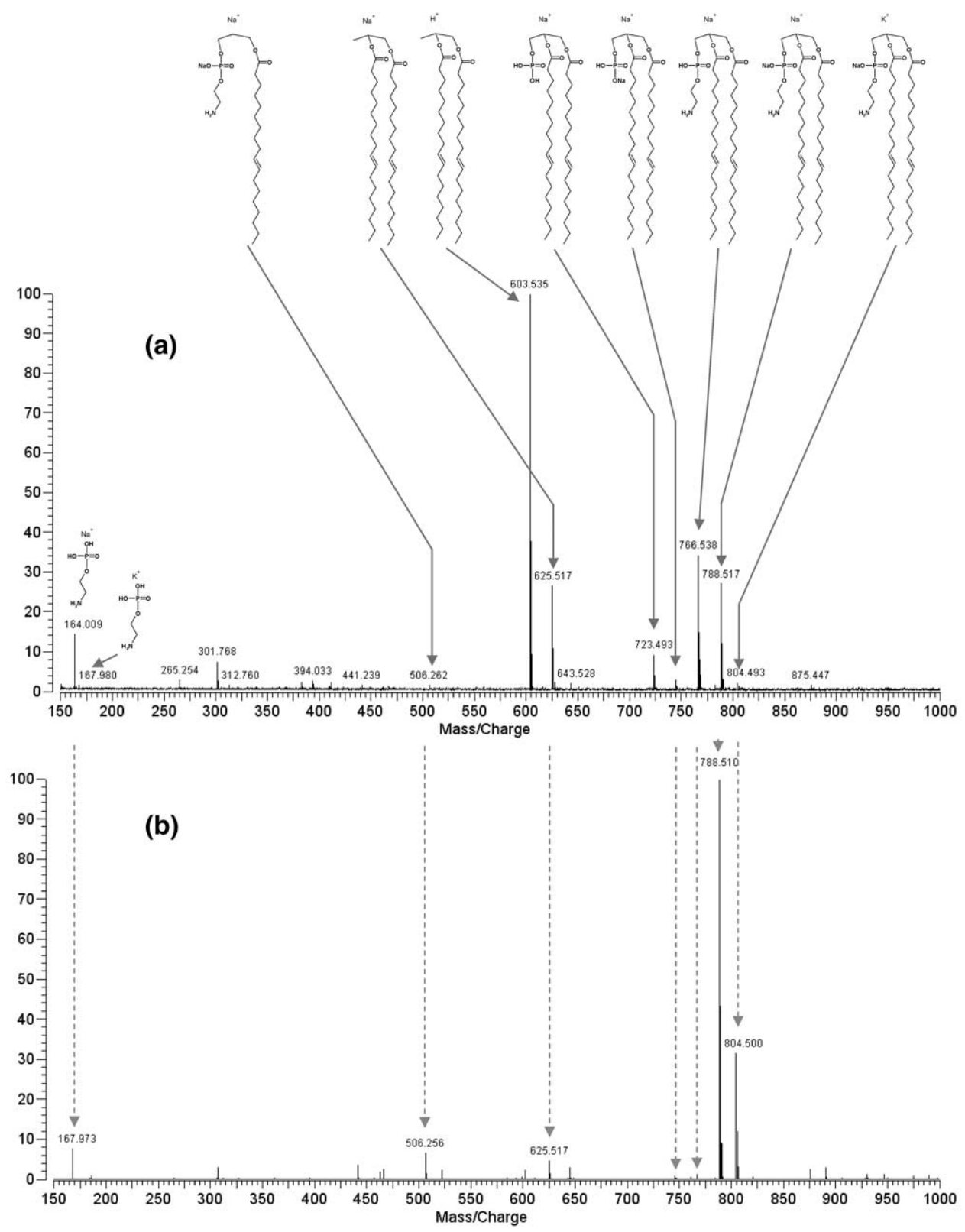

Figure 5. MALDI FTMS spectra of the phosphatidlyethanolamine, 1-2,dioleoyl-sn-glycerol-3-phosphoethanolamine, ([PE 18:1, 18:1]) analyzed using (a) DHB used as the matrix, (b) DEA-CHCA used as the matrix.

ions. Two y ions were present in the spectrum for Figure $3 \mathrm{~b}$, and the bond between Phe 8 and Gly9 did not break to produce a fragment ion. Melittin and allatostatin IV, fragment well despite being less basic peptides, producing spectra with all backbone fragments, multiple fragment ions, and ions with loss of amino acid functional groups. Extending the time between ion trapping and ion detection results in near-identical behavior for ions of each of the other peptides studied; extensive fragmentation at trapping times longer than $1 \mathrm{~s}$ followed by increasingly smaller fragments as the delay before ion excitation is extended. As somewhat expected, this trend is observed for ions produced from oligonucleotides as well.
MALDI TOF negative ion mode analysis of the oligonucleotide 5'-GGATTC-3' reveals abundant ion signals similar in response to protein analytes used with ILMs. Samples desorbed with ILMs are observed with an increase in signal intensity, again with no indication of a sample "hot spot". Only a minor number and low abundance of fragments are observed for ILMs incorporating DHB and CHCA. Previous reports of oligonucleotide analysis using MALDI FTMS required the use of 3-hydroxypicolinic acid (HPA), Figure 4a, to observe the parent ion with minimal fragmentation [27]. Oligonucleotides, consequently, follow similar behaviors to peptides for metastable decay through MALDI FTMS analysis 
using ionic liquid matrices, as well as for DHB and CHCA. The oligonucleotide used in this study produced a significant amount of fragment ions using ILMs. Here, only a few fragments are predictable and commonly observed metastable fragment ions. Furthermore, the number of peaks that could not be assigned in the mass spectra are greater in number than that for peptides, including a few of the most abundant ions detected. MALDI FTMS negative ion spectra of the oligonucleotide $5^{\prime}$-GGATTC-3' are represented in Figure 4, detailing the overwhelming abundance of fragment ions using A-CHCA and DEA-CHCA as matrices. MALDI FTMS analysis using nBA-CHCA and DHB produce spectra very similar to Figure $4 \mathrm{c}$, however the parent ion is present only in low abundance (vide infra). Furthermore, a proton to a hydroxyl or ethyl group on the oligonucleotide are apparently substituted with an alkali metal cation; sodium, potassium, or cesium. This is only evident after predicted fragments are calculated to be substituted with more than one alkali metal cation, in which case the number of assignable peaks increases. This may provide a small insight into the mechanism of fragmentation promoted by ILMs. The breakdown of peptides into smaller fragments obviously does not occur on the MALDI surface; otherwise, significant fragmentation would be observable in MALDI TOF spectra.

Phospholipids respond remarkably different to MALDI FTMS analysis using ILMs than do peptides and oligonucleotides. Figure $5 \mathrm{a}$ is a typical MALDI FTMS spectrum of phosphatidylethanolamine using DHB as the matrix; a significant amount of fragmentation is observable. The ethyl amine portion of the phosphate functional group, the entire phosphate functional group, and a long-chain ester are observed to have dissociated to leave a stable fragment ion. The parent ion is at best $35 \%$ relative abundance to the most abundant fragment ion. This observation is additionally substantiated by previous MALDI TOF reports [28, 29]. Figure $5 b$ is that of a typical MALDI FTMS spectrum of phosphatidylethanolamine using nBA-CHCA as the matrix. Fragmentation of the phospholipid has been reduced to less than $10 \%$ relative abundance to that of the parent ion. Again, this observation agrees with recent literature [24]; the observation that the overall reduction in metastable decay products for phospholipids holds even when the duration of trapping is extended for as long as $2 \mathrm{~min}$ before excitation, as in the example for peptides and oligonucleotides. There is clearly a mechanism for biopolymer fragmentation by ILMs that does not apply to smaller biomolecules such as phospholipids.

\section{Conclusions}

Ionic liquid matrices provide increased signal intensities, better spot to spot reproducibility over their traditional solid analogs, and retain the ability to be used for a wide variety of biological compounds, both with MALDI TOF and FTMS. This contrasts with a previous report where decreased sensitivity was observed [22]. However, for biopolymers such as peptides and oligonucleotides, use of ILMs induces a significant amount of slow metastable decay. The fragmentation patterns observed here are unpredictable, and may confound the identification of unknowns. Furthermore, displacing the matrix proton with an organic base, producing an ionic liquid matrix, has greatly promoted the substitution of group one alkali metal ions with, presumably, any free hydroxyl or carboxylic proton on the analyte. Fragmentation for peptides and oligonucleotides using ILMs occurs with relative ease, while the process for phospholipids appears to be the opposite. It is unclear why phospholipids are stabilized through the use of ILMs. It is hypothesized that the absence of the mobile proton of the phosphate group, which is being replaced by an alkali metal, and ionization by alkali metal addition, may prevent gas-phase fragmentation based on an entropy requirement. Extending the time between ion trapping and ion detection results in near identical behavior for protein derived ions and oligonucleotides, with extensive fragmentation that increases as the delay to ion excitation is increased. Furthermore, major spectral peaks for oligonucleotides could not be assigned to predictable fragment ions, presenting a problem for ILM use in analysis of unknowns. The suppression of fragmentation for phospholipids may aid research in the area of lipidomics.

\section{Acknowledgments}

The authors gratefully acknowledge support from the National Science Foundation grant CHE-04-55,134.

\section{References}

1. Tanaka, K.; Waki, H.; Ido, Y.; Akita, S.; Yoshida, Y. Protein and Polymer Analysis up to $m / z 100,000$ by Laser Ionization Timeof-Flight Mass Spectrometry. Rapid Commun. Mass Spectrom. 1988, 2, 151-153.

2. Karas, M.; Hillenkamp, F. Laser Desorption Ionization of Proteins with Molecular Masses Exceeding 10,000 Daltons. Anal. Biochem. 1988, 60, 2299-2301.

3. Holland, R. D.; Rafii, F.; Heinze, T. M.; Sutherland, J. B.; Voorhees, K. J.; Lay, J. O. MALDI TOF Mass Spectrometric Detection of Bacterial Biomarker Proteins Isolated from Contaminated Water, Lettuce, and Cotton Cloth. Rapid Commun. Mass Spectrom. 2000, 14, 911-917.

4. Caprioli, R. M.; Farmer, T. B.; Gile, J. Molecular Imaging of Biological Samples: Localization of Peptides and Proteins Using MALDI-TOF MS. Anal. Chem. 1997, 69, 4751-4760.

5. Easterling, M. L.; Colangelo, C. M.; Scott, R. A.; Amster, J. I. Monitoring Protein Expression in Whole Bacterial Cells with MALDI TOF Mass Spectrometry. Anal. Chem. 1998, 70, 27042709.

6. Ho, Y. P.; Fenselau, C. Applications of 1.06-um IR Laser Desorption on a Fourier Transform Mass Spectrometer. Anal. Chem. 1998, 70, 4890-4895. 
7. Chaurand, P.; Stoeckli, M.; Caprioli, R. M. Direct Profiling of Proteins in Biological Tissue Sections by MALDI Mass Spectrometry. Anal. Chem. 1999, 71, 5263-5270.

8. Holland, R. D.; Duffy, C. R.; Rafii, F.; Sutherland, J. B.; Heinze, T. M.; Holder, C. L.; Voorhees, K. J.; Lay, J. O. Identification of Bacterial Proteins Observed in MALDI TOF Mass Spectra from Whole Cells. Anal. Chem. 1999, 71, 3226-3230.

9. Fenselau, C.; Demirev, P. A. Characterization of Intact Microorganisms by MALDI Mass Spectrometry. Mass Spectrom. Rev. 2001, 20, 157-171.

10. Park, Z. Y.; Russell, D. H. Identification of Individual Proteins in Complex Protein Mixtures by High-Resolution, High-MassAccuracy MALDI TOF-Mass Spectrometry Analysis of InSolution Thermal Denaturation/Enzymatic Digestion. Anal. Chem. 2001, 73, 2558-2564.

11. Ryzhov, V.; Fenselau, C. Characterization of the Protein Subset Desorbed by MALDI from Whole Bacterial Cells. Anal. Chem. 2001, 73, 746-750.

12. Jones, J. J.; Stump, M. J.; Fleming, R. C.; Lay, J. O.; Wilkins, C. L. Investigation of MALDI-TOF and FT-MS Techniques for Analysis of Escherichia coli Whole Cells. Anal. Chem. 2003, 75, 1340-1347.

13. Stump, M. J.; Jones, J. J.; Fleming, R. C.; Lay, J. O.; Wilkins, C. L. Use of Double-Depleted ${ }^{13} \mathrm{C}$ and ${ }^{15} \mathrm{~N}$ Culture Media for Analysis of Whole Cell Bacteria by MALDI Time-of-Flight and Fourier Transform Mass Spectrometry. J. Am. Soc. Mass Spectrom. 2003, 14, 1306-1314.

14. Jones, J. J.; Stump, M. J.; Fleming, R. C.; Lay, J. O.; Wilkins, C. L. Strategies and Data Analysis Techniques for Lipid and Phospholipid Chemistry Elucidation by Intact Cell MALDIFTMS. J. Am. Soc. Mass Spectrom. 2004, 15, 1665-1674.

15. Jackson, S. N.; Wang, H. J.; Woods, A. S.; Ugarov, M.; Egan, T.; Schultz, J. A. Direct Tissue Analysis of Phospholipids in Rat Brain Using MALDI-TOFMS and MALDI-Ion MobilityTOFMS. J. Am. Soc. Mass. Spectrom. 2005, 16, 133-138.

16. Cooks, G. Mass Spectrometry Sampling Under Ambient Conditions with Desorption Electrospray Ionization. Science 2004, 306, 471-473.

17. Karas, M.; Kruger, R. Ion Formation in MALDI: The Cluster Ionization Mechanism. Chem. Rev. 2003, 103, 427-493.

18. Horneffer, V.; Reichelt, R.; Strupat, K. Protein Incorporation into MALDI-Matrix Crystals Investigated by High Resolution Field Emission Scanning Electron Microscopy. Int. J. Mass Spectrom. 2003, 226, 117-131.
19. Barber, M.; Bordoli, R. S.; Sedgwick, R. D.; Tyler, A. N. Fast Atom Bombardment of Solids (FAB): A new Ion Source for Mass Spectrometry. J. Chem. Soc. Chem. Commun. 1981, 325-327.

20. Armstrong, D.; Zhang, L.-K.; He, L.; Gross, M. Ionic Liquids as Matrixes for Matrix-Assisted Laser Desorption/Ionization Mass Spectrometry. Anal. Chem. 2001, 73, 3679-3686.

21. Carda-Broch, S.; Berthod, A.; Armstrong, D. W. Ionic Matrices for Matrix-Assisted Laser Desorption/Ionization Time-ofFlight Detection of DNA Oligomers. Rapid Commun. Mass Spectrom. 2003, 17, 553-560.

22. Mank, M.; Stahl, B.; Boehm, G. 2,5-Dihydroxybenzoic Acid Butylamine and Other Ionic Liquid Matrixes for Enhanced MALDI-MS Analysis of Biomolecules. Anal. Biochem. 2004, 76, 2938-2950.

23. Zabet-Moghaddam, M.; Heinzle, E.; Tholey, A. Qualitative and Quantitative Analysis of Low Molecular Weight Compounds by Ultraviolet Matrix-Assisted Laser Desorption/ Ionization Mass Spectrometry Using Ionic Liquid Matrices. Rapid Commun. Mass Spectrom. 2004, 18, 141-148.

24. Li, Y. L.; Hsu, F.; Gross, M. L. Ionic-Liquid Matrices for Improved Analysis of Phospholipids by MALDI-TOF Mass Spectrometry. J. Am. Soc. Mass Spectrom. 2005, 16, 679-682.

25. Senko, W. M.; Speir, J. P.; McLafferty, F. Collisional Activation of Large Multiply Charged Ions Using Fourier Transform Mass Spectrometry. Anal. Biochem. 1994, 66, 2801-2808.

26. Little, D. P.; Speir, J. P.; Senko, M. W.; O'Connor, P. B.; McLafferty, F. W. Infrared Multiphoton Dissociation of Large Multiply Charged Ions for Biomolecule Sequencing. Anal. Biochem. 1994, 66, 2809-2815.

27. Li, Y.; Tang, K.; Little, D. P.; Koster, H.; Hunter, R. L.; Robert T. McIver, J. High-Resolution MALDI Fourier Transform Mass Spectrometry of Oligonucleotides. Anal. Biochem. 1996, 68, 2090-2096.

28. Zabrouskov, V.; Al-Saad, K. A.; Siems, W. F.; Hill, H. H.; Knowles, R. Analysis of Plant Phosphatidylcholine by MatrixAssisted Laser Desorption/Ionization Time-of-Flight Mass Spectrometry. Rapid Commun. Mass Spectrom. 2001, 15, 935940.

29. Al-Saad, K. A.; Zabrouskov, V.; Siems, W. F.; Knowles, N. R.; Hannan, R. M.; Hill, H. H. Matrix-Assisted Laser Desorption/ Ionization Time-of-Flight Mass Spectrometry of Lipids: Ionization and Prompt Fragmentation Patterns. Rapid Commun. Mass Spectrom. 2003, 17, 87-96. 